\title{
Prostate tumor eccentricity predicts Gleason score better than prostate tumor volume
}

\author{
Rulon Mayer ${ }^{1,2}$, Charles B. Simone $\mathrm{II}^{3}$, Baris Turkbey ${ }^{4}$, Peter Choyke ${ }^{4}$ \\ ${ }^{1}$ University of Pennsylvania, Philadelphia, PA, USA; ${ }^{2}$ Oncoscore, Garrett Park, MD, USA; ${ }^{3}$ New York Proton Center, New York, NY, USA; \\ ${ }^{4}$ National Institutes of Health, Bethesda, MD, USA
}

Contributions: (I) Conception and design: R Mayer; (II) Administrative support: R Mayer, CB Simone, P Choyke; (III) Provision of study materials or patients: B Turkbey; P Choyke; (IV) Collection and assembly of data: R Mayer; (V) Data analysis and interpretation: R Mayer; (VI) Manuscript writing: All authors; (VII) Final approval of manuscript: All authors.

Correspondence to: Rulon Mayer, MD. University of Pennsylvania, Philadelphia, PA 19104, USA; OncoScore, 10702 Keswick St., Garrett Park, MD 20896, USA. Email: mayerru@yahoo.com.

Background: Prostate tumor volume predicts biochemical recurrence, metastases, and tumor proliferation. A recent study showed that prostate tumor eccentricity (elongation or roundness) correlated with Gleason score. No studies examined the relationship among the prostate tumor's shape, volume, and potential aggressiveness.

Methods: Of the 26 patients that were analyzed, 18 had volumes $>1$ cc for the histology-based study, and 25 took up contrast material for the MRI portion of this study. This retrospective study quantitatively compared tumor eccentricity and volume measurements from pathology assessment sectioned wholemount prostates and multi-parametric MRI to Gleason scores. Multi-parametric MRI (T1, T2, diffusion, dynamic contrastenhanced images) were resized, translated, and stitched to form spatially registered multi-parametric cubes. Multi-parametric signatures that characterize prostate tumors were inserted into a target detection algorithm (Adaptive Cosine Estimator, ACE). Various detection thresholds were applied to discriminate tumor from normal tissue. Pixel-based blobbing, and labeling were applied to digitized pathology slides and threshold ACE images. Tumor volumes were measured by counting voxels within the blob. Eccentricity calculation used moments of inertia from the blobs.

Results: From wholemount prostatectomy slides, fitting two sets of independent variables, prostate tumor eccentricity (largest blob eccentricity, weighted eccentricity, filtered weighted eccentricity) and tumor volume (largest blob volume, average blob volume, filtered average blob volume) to Gleason score in a multivariate analysis, yields correlation coefficient $\mathrm{R}=0.798$ to 0.879 with $\mathrm{P}<0.01$. The eccentricity t-statistic exceeded the volume t-statistic. Fitting histology-based total prostate tumor volume against Gleason score yields $\mathrm{R}=0.498, \mathrm{P}=0.0098$. From multi-parametric MRI, the correlation coefficient $\mathrm{R}$ between the Gleason score and the largest blob eccentricity for varying thresholds $(0.30$ to 0.55$)$ ranged from -0.51 to $-0.672(\mathrm{P}<0.01)$. For varying thresholds ( 0.60 to 0.80 ) for MRI detection, the $\mathrm{R}$ between the largest blob volume eccentricity against the Gleason score ranged from 0.46 to $0.50(\mathrm{P}<0.03)$. Combining tumor eccentricity and tumor volume in multivariate analysis failed to increase Gleason score prediction.

Conclusions: Prostate tumor eccentricity, determined by histology or MRI, more accurately predicted Gleason score than prostate tumor volume. Combining tumor eccentricity with volume from histologybased analysis enhanced Gleason score prediction, unlike MRI.

Keywords: Tumor morphology; prostate cancer (PCa); multi-parametric MRI; Gleason score; histology of wholemount prostatectomy

Submitted May 05, 2021. Accepted for publication Sep 03, 2021.

doi: 10.21037/qims-21-466

View this article at: https://dx.doi.org/10.21037/qims-21-466 


\section{Introduction}

The high prevalence and varying natural history of prostate cancer $(\mathrm{PCa})$ motivates the need to distinguish indolent from potentially lethal PCa. Patient management for PCa requires accurate identification at the time of $\mathrm{PCa}$ diagnosis for whom treatment is indicated and to predict cancer progression. Traditionally, clinical factors (1) stratify PCa patients and guide the assessed risk of cancer progression and need for treatment. More recently, molecular markers (2) in tumors and circulation have influenced disease management. Noninvasive diagnostic imaging (3), especially MRI and magnetic resonance spectroscopic imaging, has improved in recent years and is gaining widespread acceptance for aiding PCa diagnosis, tumor localization, staging, assessment of tumor aggressiveness, and treatment planning. Investigators have used nomogram and neural network modeling to predict organ-confined PCa (4), clinically significant disease (5), and risk of biochemical recurrence after radical prostatectomy (6).

The Gleason score grading system, first described in the 1960s, characterizes prostate tumor architecture and morphology. Currently a standard metric for assessing $\mathrm{PCa}$, Gleason score is also the strongest clinical predictor of PCa progression (7). In addition, studies (8-12) show that prostate tumor volume correlates with Gleason Score grade, stage, disease progression and biochemical-free survival after radical prostatectomy, probability of vascular invasion, involvement of seminal vesicles, and development of distant metastasis. For example, small tumors $\left(<0.5 \mathrm{~cm}^{3}\right)$ display limited evidence of tumor aggressiveness. However, metastasis is highly likely when tumor volume exceeds $12 \mathrm{~cm}^{3}$ but less likely for smaller tumor volumes $\left(<4 \mathrm{~cm}^{3}\right)(12)$. Others reported that tumor size is related to the risk of relapse following radical prostatectomy (13) and prostatespecific antigen (PSA) progression (14).

Tumor shape, whether spherical or elongated into an ellipsoid and quantified by a metric called eccentricity (15), is not usually measured nor currently considered in staging for PCa, unlike breast $(16,17)$, lung $(18,19)$, and skin $(20,21)$ cancers. Recently, however, a study (15) showed that spherical prostate adenocarcinoma shape, rather than elongated, shows a propensity for higher Gleason score and presumed greater aggressiveness. This important finding follows the behavior of lung and breast adenocarcinomas but markedly differs from other primary tumor types.

Conventional cancer staging and scoring through biopsies $(22,23)$ suffer from significant inter-observer variability and/or potential for sampling errors (24) that can lead to false negatives or underestimate the severity of the disease. Moreover, the quality of MRI radiological interpretations can depend on the skill and experience of the reader. For example, the PI-RADS (25) assessment of tumor aggressiveness requires experienced and specially trained radiologists.

This manuscript describes the relationship between Gleason score, an indicator for patient outcome for this study, and tumor volume (Vol) and tumor eccentricity (Ecc). This study examined the individual correlation and the combined correlation of Vol and Ecc with Gleason score. Due to relative dearth of information regarding tumor eccentricity and Gleason score, a part of this study examined pathology assessment of histology slices to provide baseline information. Future studies may find the added input from histology, alone, may benefit clinical management. However, this study focused on non-invasive MP-MRI. Three metrics (Vol, Ecc, Gleason score) may be inferred non-invasively $(15,26,27)$ by assessing spatially registered multi-parametric (MP-MRI) hypercubes with minimal involvement of clinical evaluation and intervention of radiologists, reduced patient discomfort, and more reliable assessment of patient conditions. However, in this study, Gleason score derived from histology is treated as the standard reference and is the dependent variable in the univariate and multivariate fittings.

We present the following article in accordance with the MDAR checklist (available at https://dx.doi.org/10.21037/ qims-21-466).

\section{Methods}

\section{Overall description of histology and MP-MRI determination}

This study followed two tracks (Figure 1) $(15,27)$ to determine the relationship between prostate tumor morphology (eccentricity), tumor volume, and Gleason score. One track examined tumors outlined on histology slides derived from wholemount prostatectomy from tumors delineated by a pathologist. The other track computed eccentricity and tumor volume from Adaptive Cosine Estimator (ACE) applied to spatially registered multi-parametric MRI. The analysis studied 25 of 26 consecutive patients who previously underwent radical prostatectomy, Gleason score grading by pathologists and denoted as the dependent variable (solid red arrow in 

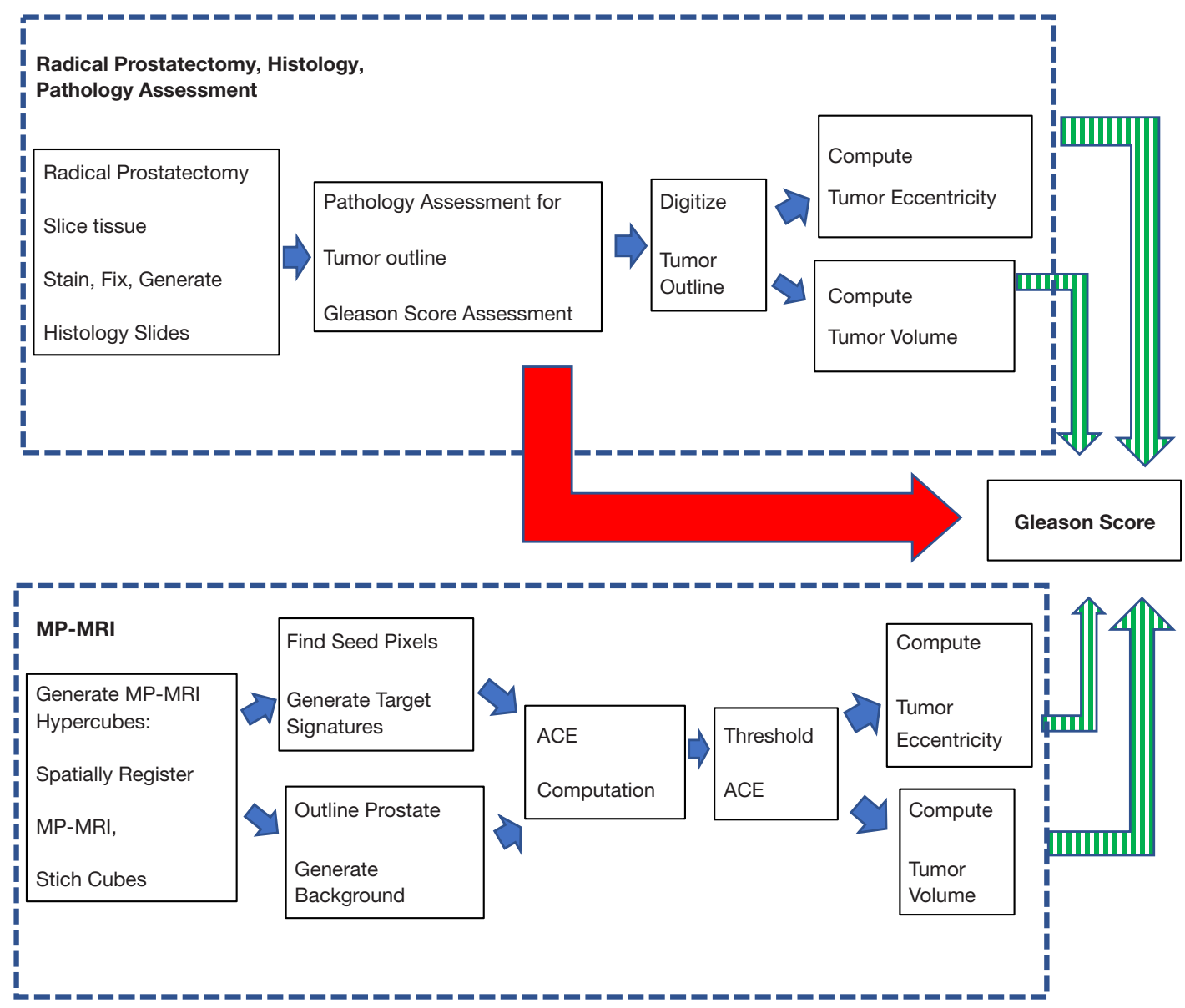

Figure 1 Schematic showing the overall procedure for generating tumor eccentricity and tumor volume from blobs derived from histology of wholemount prostatectomy specimens and ACE processing applied to multi-parametric MRI. Blob eccentricity and volumes compared to Gleason scoring from histology (striped arrow). ACE, Adaptive Cosine Estimator.

Figure 1). One of the 26 patients did not display contrast uptake in the MRI dynamic contrast enhancement images and was not analyzed. The calculated eccentricity (15) in both tracks were derived from computing the moment of inertia for all pixels residing in the blob. The tumor volume (27) in both tracks were determined by summing the voxels in each blob. The tumor eccentricity and tumor volume were then compared (striped arrows in Figure 1) to the Gleason score of the tumor.

\section{Study design and population}

Patient data from prostate tumor MRI and histology from wholemount prostatectomy specimens were collected and stored with The Cancer Imaging Archive (TCIA) $(28,29)$ affiliated with The National Institutes of Health (NIH). The study was conducted in accordance with the Declaration of Helsinki (as revised in 2013). This retrospectively designed, single institution study was approved by the NIH Institutional Review Board, and was compliant with the Health Insurance Portability and Accountability Act. And individual consent for this retrospective analysis was waived. A total of 26 consecutive patients enrolled in the study between July 2008 and July 2009. All patients had biopsy proven adenocarcinoma of the prostate. Median patient age was 60 years (range, $49-75$ years). Median PSA was $5.8 \mathrm{ng} / \mathrm{mL}$ (range, $2.3-23.7 \mathrm{ng} / \mathrm{mL}$ ). Median Gleason score was 7 (range, 6-9). Eighteen of the 26 patients had tumor size $>1$ cc. No restrictions were placed on tumor location within the prostate. A robotic assisted radical prostatectomy was performed with a median time of 60 days (range, 3-180 days) after the MRI without any intervening treatment. 


\section{Whole mount prostatectomy and bistology}

Three dimensional (3D) models of each prostate $(30,31)$ were generated by segmenting the prostate capsule on in vivo triplane T2W MRI, and extracting from the highresolution 3D surfaces. Each mold was designed using commercially available 3D computer aided design software. The design incorporated the deformation of the endorectal coil. A 3D printer (Dimension Elite 3D printer, Stratasys, Inc.) deposited styrene to fabricate each mold. Following radical prostatectomy, the specimen was fixed at room temperature in formalin for 2 to 24 hours. The specimen was then placed in the customized 3D mold and sliced in sections with a separation of $6 \mathrm{~mm}$. in the axial direction. The whole mount histopathology patient specimens were sectioned in the customized mold for histopathology that corresponded to the axial plane of the MRI sections. Two experienced pathologists independently, and blinded from MRI analysis, mapped the slides for individual tumor foci, dimensions, and Gleason scores.

The determination of a tumor's morphology is taken from histology slides of sectioned wholemount prostatectomy. A pathologist marks the tumor and the marked tumors are digitally traced. The eccentricity and volume for the blobs are computed using Eqs. [1-4].

\section{MRI}

The MRI collection is composed of Diffusion Weighted Images (DWI), Dynamic Contrast Enhanced (DCE), and structural (T1, T2) images. The pulse sequences were described in earlier studies (30-32). These studies (30-32) were performed using a combination of an endorectal coil (BPX-30, Medrad) tuned to $127.8 \mathrm{MHz}$ and a 16-channel cardiac coil (SENSE, Philips Medical Systems). The MRI used a 3 Tesla (T) magnet (Achieva, Philips Medical Systems). Prior bowel preparation was not needed. Standard methods were used to insert the endorectal coil. The MRI protocol included triplanar T2W turbo spin echo, DW MRI, 3DMR point resolved spectroscopy, and axial pre-contrast T1-weighted axial 3D fast field echo DCE MRI sequences, and their detailed sequence parameters were defined in a prior study (32). The mean interval between MRI and radical prostatectomy was 60 days (range, 3-180 days).

\section{Image processing, pre-analysis}

Lesions are distinguished from normal tissues by the presence of elevated vasculature that feeds the rapidly growing tumor. DCE are time series images that display the time evolution of contrast material over several hundred seconds after its injection and shows uptake in the tissues. The vasculature is porous to contrast material that enters the tumor's extravascular space (but not the cells). The vasculature of prostate tumors can fill and empty of MRI contrast material more quickly than the normal prostate organ. By analyzing the DCE and exploiting the unique tumor physiology, a portion of tumors maybe identified. A simple two compartment model $(33,34)$ describes the tracer concentration in the tissue that supplies and empties through the tumor vasculature. The model is used to create the washout $\mathrm{k}_{\mathrm{ep}}$ image.

The MRI images were digitally resized $(15,26,27)$ to $1 \mathrm{~mm}$ resolution in the transverse direction. In the superiorinferior direction, the slices were resized to $6 \mathrm{~mm}$ spacing based on the patient's table position. Fine-tuned rigid registration (minor transverse translation, resizing) was applied between the structural, diffusion, and DCE due to the short time intervals between scans ( $<20$ minutes). Individual slices scaled, translated, resliced and spatially were thereby registered at the pixel level to create a "cube". Multiple axial cubes in three dimensions were "stitched" together by sequentially connecting them together into a narrow three-dimensional image. Four dimensions (threedimensional body volume plus the fourth dimension composed of MRI modalities) object is re-expressed as a three-dimensional mosaicked cube. Each voxel is treated as a vector composed of MRI modality rather than a scalar value. Multispectral MRI data contain 7-components $(15,26,27)$ (T1 (pre contrast), T1 (maximum contrast), T2, ADC, DWI-High B $\left(B=1,000 \mathrm{~s} / \mathrm{mm}^{2}\right)$, Washout or $\mathrm{k}_{\text {ep }}$ from DCE).

\section{$A C E$}

The multispectral Supervised Target Detection (STDA) methods (35-37), specifically the ACE (Eq. [1]), were transferred to this medical application $(15,26,27)$ and applied to spatially registered MP-MRI. The algorithm (ACE) uses in-scene multispectral tumor signatures (for tumor). $\mathrm{S}$ the target (tumor) signature is a 7-component vectors [DWI, T1, T2, ADC, DCE for this study $(15,26,27)] . S$, the in-scene tumor signature, is selected from yellow voxels in a three-color display of the spatiallyregistered MP-MRI (red is Washout, green is DWI, high-B, blue is $\mathrm{ADC})(15,26,27)$. $\mathrm{S}$ vector, with component $\mathrm{S}_{\mathrm{q}}$, is the 
mean of $\mathrm{T}$ target vector-voxels $\mathrm{x}_{\mathrm{p}, \mathrm{q}}$ summed over $\mathrm{p}$ target voxels (identified as yellow) (Eq. [1]). $\mathrm{m}$ is the background (normal prostate) 7 component vector. $\mathrm{CM}$ is the covariance (7x7) matrix for the background. The background voxels needed for $\mathrm{m}$ and $\mathrm{CM}$ were taken from digitally outlining the prostate on the spatially-registered MP-MRI $(15,26,27)$. ACE in Eq. [1] is a matrix multilplication with the superscript -1 is a matrix inversion and $T$ is the transpose.

$$
\begin{aligned}
& S_{q}=\frac{1}{T} \sum_{p=1}^{T} x_{p . q}, \\
& A C E\left(x_{i}\right)=\frac{(S-m)^{T} C M^{-1}\left(x_{i}-m\right)}{\sqrt{\left[(S-m)^{T} C M^{-1}(S-m)\right]\left[\left(x_{i}-m\right)^{T} C M^{-1}\left(x_{i}-m\right)\right]}}
\end{aligned}
$$

$\operatorname{ACE}\left(\mathrm{x}_{\mathrm{i}}\right)$ is the cosine between the "whitened" test vector $\mathrm{x}_{\mathrm{i}}$ for voxel $\mathrm{i}$ and the "whitened" target signature S (Eq. [1]) in the "whitened space" $(26,37)$. To compute the background statistics, the prostate image is manually outlined for all slices to generate an image mask and restrict computations to the prostate volume. The signature $\mathrm{S}$ is substituted into the ACE algorithm or to classify each voxel $(15,26,37)$ whether it is tumor or normal prostate tissue. ACE uses the conical hyperspace decision surface to evaluate whether a voxel is background (large angle, small cosine) or target (small angle or large cosine). ACE scores range from -1.0 to 1.0. The identification of the voxel depends on the detection threshold set by the user based on previously examined data, such as optimal correlation with a standard.

\section{Tumor volume measurements, supervised target detection}

Tumor volume from histology determination was derived directly from tumors outlined by a pathologist on slides (27). Tumor volume was given by digitally tracing the tumor for all slices, summing the number of voxels within the pathologists outline and converting the number of voxels to volume form knowing the image resolution (300 dots per inch) and the histology slice separation $(6 \mathrm{~mm})$.

The procedure for estimating the tumor volume using the supervised target detection algorithm or ACE has been previously described (27) for spatially-registered MPMRI. Detection scoring by computing ACE using in-scene signatures has been described in Section F $(15,26,27)$, and summarized through Eq. [1]. A threshold is applied to the ACE map with tumor exceeding the threshold and normal tissue assigned to ACE scores residing below the threshold. The chosen threshold in this study was from 0.40 to 0.85 assessed in 0.05 increments. The number of tumor voxels are converted to volume using the MRI spatial resolution
$(1 \mathrm{~mm} \times 1 \mathrm{~mm})$ and slice thickness $(6 \mathrm{~mm})$ to compute the single voxel volume $\left(0.006 \mathrm{~cm}^{3}\right)$. The correlation coefficient between tumor volume and Gleason scores, derived from histology $(15,26,27)$ were computed. ACE threshold was chosen based on the highest correlation with Gleason scores.

\section{Labeling and blob generation}

Blobbing and labeling (15) in computer vision aggregate neighboring pixels. The blobbing is applied to a binary image following application of a threshold to the primary image. Blobbing is based on whether the pixels form an 8-pixel connected neighborhood. In this study, binary tumor masks are generated from histology slides and from images showing the pixels that exceed a threshold of ACE target detection applied to MP-MRI hypercubes. Specifically, the value of 1(0) or "True" ("False") is associated with tumor (background) in each masked image. Each "True" pixel peruses pixels within a given neighborhood (1 pixel away) to see if they are also "True" and are, therefore, connected, collected, and labeled as a member of a blob. Blobs smaller than $<0.175 \mathrm{cc}$ are filtered out.

\section{Eccentricity calculation}

Eccentricity and volume [15] were computed for every labeled blob using custom software coded in Python 3. To compute the eccentricity, the largest axis $\mathrm{L}_{\mathrm{k}}$ and transverse moments $S_{k}$ are eigenvalues from the moment of inertia matrix I for the kth blob, where I

$$
I=\left(\begin{array}{ll}
I_{x x} & I_{x y} \\
I_{y x} & I_{y y}
\end{array}\right)
$$

and the standard defined matrix components $I_{x x}, I_{y y}, I_{x y}$, and $I_{y x}$. The eccentricity $E_{k}$ for the kth blob with a major axis $L_{k}$ and minor axis $S_{k}$ is given by

$$
E_{k}=\frac{L_{k}-S_{k}}{L_{k}}
$$

Eccentricity values $E_{k}$ range from 0 to 1 . A spherical shape has an eccentricity $E_{k}$ of 0 while a line has an eccentricity $\mathrm{E}_{\mathrm{k}}$ of 1 . Assuming density of unity, each Blob's volume $V_{k}$ is given by the image resolution $r$ and the number of pixels within each blob.

$$
V_{k}=r N=r \sum_{i=1}^{N} \frac{x_{i}}{x_{i}}
$$


and $\mathrm{r}$ is the total volumetric resolution $\left(6 \mathrm{~mm}^{3}\right.$ per voxel for MP-MRI and $0.00270 \mathrm{~mm}^{3}$ per voxel for the histology slices in this study).

The weighted eccentricity $\mathrm{W}$ is sum over B blobs, given by

$$
W=\frac{\sum_{k=1}^{B} V_{k} E_{k}}{\sum_{k=1}^{B} V_{k}}
$$

\section{Statistics and univariate and multivariate fitting}

The Gleason score derived from the pathological assessment of histology slides from prostatectomy were fitted individually through linear regression with eccentricity (Ecc) or volume (Vol). The dependent variable, Gleason score, is univariately linearly related to the independent measurements Ecc and Vol i.e.,

$$
\begin{aligned}
& G S=b_{\text {const }}+b_{E c c} * E c c+\varepsilon \\
& G S=b_{\text {const }}+b_{V o l} * V o l+\varepsilon
\end{aligned}
$$

Optimal coefficients $b_{c o n s t}, b_{E c c}$ and $b_{V o l}$ or constant, eccentricity coefficient, and volume coefficients respectively, were chosen by minimizing the error $\varepsilon$ through the leastsquares formulation. In this study, Ecc includes Eccentricity from the largest blob, eccentricity of the weighted average, and a filtered weighted average. The analysis filters out contributions from blobs smaller than $0.175 \mathrm{~cm}^{3}$ due to spatial registration errors inducing spurious detections. Vol or Volume includes the total tumor or blob Volume, average blob volume, and a filtered average blob volume that removes contributions from blobs smaller than $0.175 \mathrm{~cm}^{3}$. The Pearson correlation coefficient R, R squared (rsquared), adjusted correlation coefficient, $t$ value, $P$ value, that assess the fitness and probability that the fit departs from null fit, were computed. In addition (38-41), the quality of fit was assessed by computing the $\mathrm{F}$ value and affiliated $\mathrm{P}$ value, Akaike Information Criterion (AIC), Bayes Information Criterion (BIC).

The conditional number computed for the independent variables, eccentricity and volume measurements, found them to be independent. Testing to evaluate if the data follows normal distribution was assessed by computing the skewness and kurtosis of the distribution, Jarque-Bera (JB) metric and its affiliated probability. Correlation among the residuals in the regression were assessed through the Durbin-Watson metric. Due to the relatively small sample size of 25 , the fits were also assessed using the Leave One Out Coefficient (LOOC).

Many of the metrics discussed in the previous paragraph describe similar and overlapping assessments of the fitting and therefore yield similar results. To simplify the discussion without sacrificing a proper and meaningful evaluation of the fits, only a selected metrics will be mentioned in this manuscript. Specifically, the data tables only cite the fitted coefficients $\mathrm{b}$ with the associated computed standard error, the $t$-statistic and associated $P$ value, Pearson $R, P$ value, $\mathrm{R}^{2}$, adjusted correlation coefficient, $\mathrm{F}$ value and affiliated $\mathrm{P}$ value.

In addition to univariate fits, a multivariate fit (Eq. [8]) was applied to the Gleason score, eccentricity, and volume measurements i.e.,

$$
G S=b_{\text {const }}+b_{E c c} * E c c+b_{V o l} * V o l+\varepsilon
$$

\section{Results}

\section{Histology analysis}

Figure $2 \mathrm{~A}$ plots the histology-based eccentricity of the largest blob and the weighted eccentricity against Gleason score (Eq. [6]) for the 18 tumor volumes exceeding $1 \mathrm{cc}$. The correlation coefficient $\mathrm{R}$ and corresponding $\mathrm{P}$ value for both the largest blob and a weighted average was $R=0.70$, $\mathrm{P}=0.0005$ and $\mathrm{R}=0.68, \mathrm{P}=0.001$, respectively. For all tumor volumes, the correlation coefficient $\mathrm{R}$ and corresponding $\mathrm{P}$ value for both the largest blob and a weighted average was $\mathrm{R}=0.22, \mathrm{P}=0.28$ and $\mathrm{R}=0.19, \mathrm{P}=0.36$, respectively, demonstrating the importance of filtering out spurious blobs. Figure $2 B$ plots histology-based total volume and volume of largest blob against Gleason score (Eq. [7]). For tumor volumes exceeding $1 \mathrm{cc}$, the correlation coefficient $\mathrm{R}$ and corresponding $\mathrm{P}$ value for both the average and a total volumes was $\mathrm{R}=0.63, \mathrm{P}=0.0028$ and $\mathrm{R}=0.65, \mathrm{P}=0.0019$, respectively. For tumor volumes exceeding $1 \mathrm{cc}$, Figure $2 \mathrm{C}$ plots Gleason score data against the multivariate fit (Eq. [8]) that uses the histology-based eccentricity from the largest blob plus the histology-based total volumes for Gleason score. A univariate fit between the Gleason score data and multivariate fit to the Gleason score results in the correlation coefficient and corresponding $\mathrm{P}$ value $(\mathrm{R}=0.879$, $\mathrm{P}<1 \mathrm{E}-05)$, respectively.

Table 1 summarizes metrics for assessing the multivariate 
A

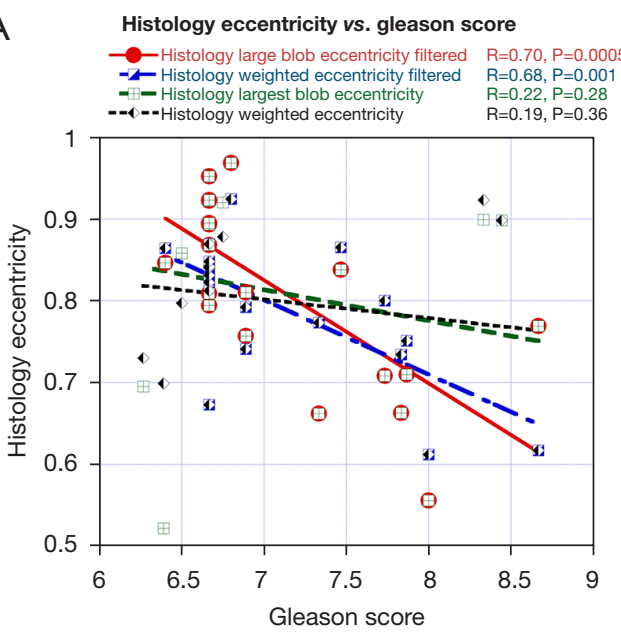

B

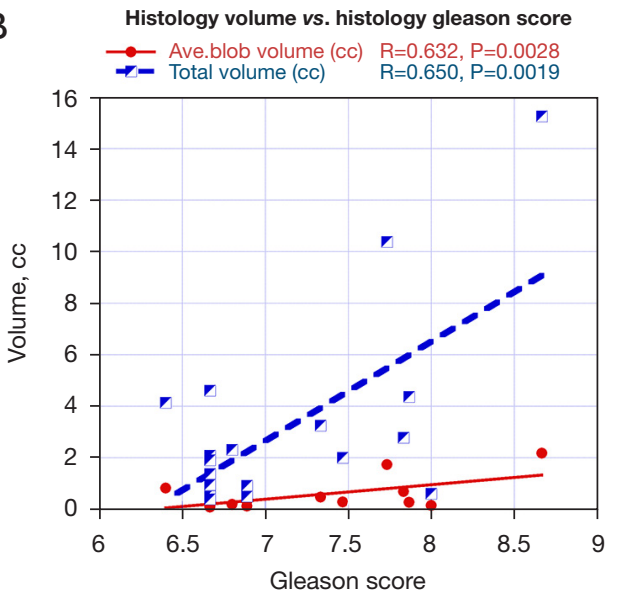

C Gleason Score, Data vs. Multivariable Linear Regression Fit Eccentricity (Blob Max) + Total Volume $\mathrm{R}=0.879, \mathrm{P}<1 \mathrm{E}-05$

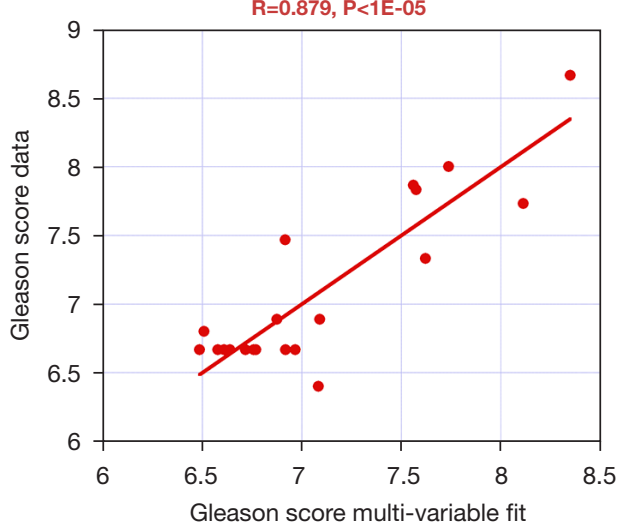

Figure 2 Scatterplots and show correlation coefficients, $\mathrm{P}$ values for histology-based Gleason score against histology-derived eccentricity, volume, and multivariable fits for 18 patients. (A) Plots histology eccentricity of largest blob and weighted eccentricity against Gleason score; (B) plots histology total volume and volume of largest blob against Gleason score; (C) plots multivariate fit for Gleason score against data for Gleason score.

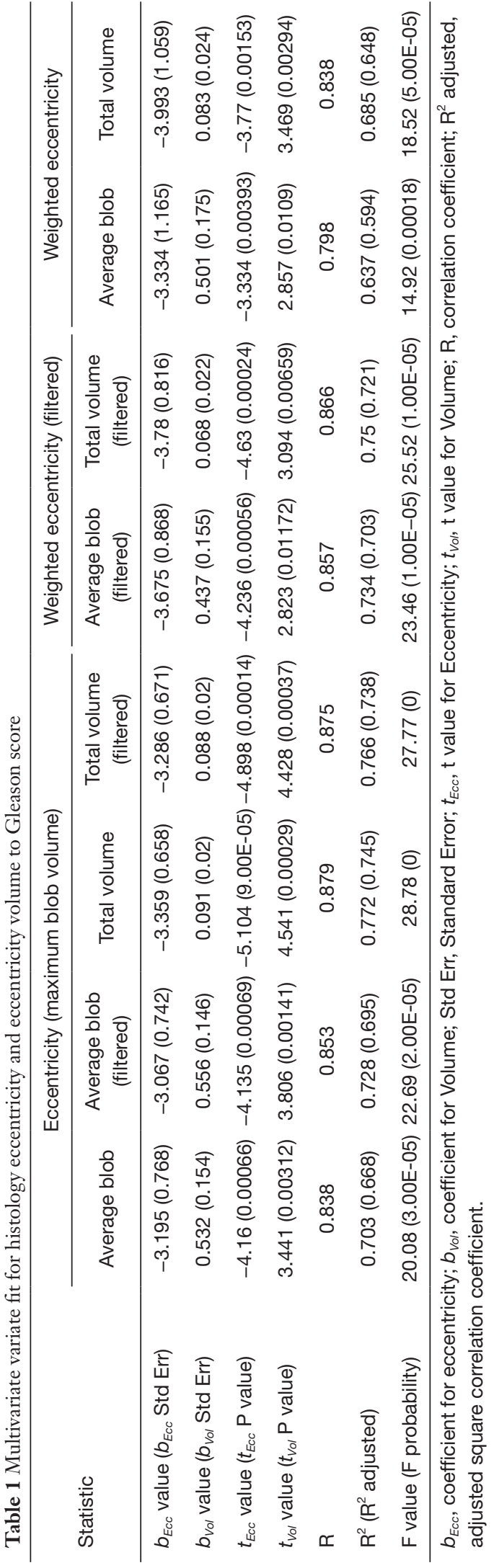


Table 2 Metrics for univariate fits to Gleason Score using MP-MRI largest blob eccentricity ACE thresholds 0.35 to 0.55

\begin{tabular}{lccccc}
\hline Statistic & $\begin{array}{c}0.35 \text { ACE max blob } \\
\text { eccentricity }\end{array}$ & $\begin{array}{c}0.40 \text { ACE max blob } \\
\text { eccentricity }\end{array}$ & $\begin{array}{c}0.45 \text { ACE max blob } \\
\text { eccentricity }\end{array}$ & $\begin{array}{c}0.50 \text { ACE max blob } \\
\text { eccentricity }\end{array}$ & $\begin{array}{c}0.55 \text { ACE max blob } \\
\text { eccentricity }\end{array}$ \\
\hline$b_{E C C}(\mathrm{P}$ value $)$ & $-2.628(0.736)$ & $-2.949(0.704)$ & $-2.494(0.632)$ & $-2.491(0.649)$ & $-2.614(0.6)$ \\
$t_{E c C}(\mathrm{P}$ value $)$ & $-2.823(0.00964)$ & $-3.568(0.00163)$ & $-4.191(0.00035)$ & $-3.947(0.00064)$ & $-4.358(0.00023)$ \\
$R$ & -0.507 & -0.597 & -0.658 & -0.636 & -0.672 \\
$R^{2}\left(R^{2}\right.$ adjusted $)$ & $0.257(0.225)$ & $0.356(0.328)$ & $0.433(0.408)$ & $0.404(0.378)$ & $0.452(0.429)$ \\
F statistic $(F$ prob) & $7.97(0.00964)$ & $12.731(0.00163)$ & $17.567(0.00035)$ & $15.58(0.00064)$ & $18.995(0.00023)$ \\
\hline
\end{tabular}

$\mathrm{ACE}$, algorithm; $b_{E c c}$, coefficient for eccentricity; Std Err, standard error; $t_{E c c}$, $t$ value for eccentricity; $\mathrm{R}$, correlation coefficient; ACE max blob eccentricity, eccentricity for largest blob; $R^{2}$, squared correlation coefficient; $R^{2}$ adjusted, adjusted square correlation coefficient.

fits (Eq. [8]) for histology-based eccentricity and volume measurements to Gleason score. The two sets of independent variables use combinations of prostate tumor eccentricity and volume. Options for $b_{E c c}$ include the eccentricity for the largest blob, weighted average for the eccentricity and filtered version of the weighted eccentricity that exclude blob volumes smaller than $0.175 \mathrm{~cm}^{3}$. Options for $b_{V l}$ include the average blob volume, the filtered average blob volume, total volume, and filtered total volume. The fitted coefficients for the eccentricity and volume $b_{E c c}$ and $b_{V o l}$ are shown along with the standard error for each parameter associated with the fit. The $b_{E c c}$ is negative, demonstrating that larger Gleason score is associated with smaller and more spherical tumors. The $b_{V o l}$ is positive, confirming that larger tumors are associated with greater Gleason score. The $\mathrm{t}$ statistics for eccentricity $t_{E c c}$ and volume and $t_{V o l}$ along with $\mathrm{P}$ values are displayed in Table 1. The magnitude of the $\mathrm{t}$ value $t_{E c c}$ always exceeds $\mathrm{t}$ value $t_{V o l}$ for all pairs of independent variables. A specific example taken from Table 1: eccentricity (maximum blob volume) yields $t_{E c c}=-4.16$ and Average Blob Volume $t_{V o l}=3.441$.

Correlation coefficient $\mathrm{R}, \mathrm{R}^{2}$, adjusted $\mathrm{R}^{2}, \mathrm{~F}$ statistic and $P$ value summarize the overall multivariate fit for histologybased measurements to Gleason score (Eq. [8]).

In Table 1, analysis of histology slides from wholemount prostatectomy specimens finds the correlation coefficient $\mathrm{R}$ and $\mathrm{P}$ value from fitting two sets of independent variates in a multivariate analysis (Eq. [8]) for Gleason score ranged from R 0.798 to 0.879 and $\mathrm{P}$ value 0.000018 to $<0.00001$, respectively. The t-statistic for the eccentricity exceeded those from volume measurements. This histology analysis processed 18 patients whose total tumor volume exceeded $1.0 \mathrm{cc}$.

The correlation coefficient and $\mathrm{P}$ value from fitting total prostate tumor volume derived from histology slides and Gleason score are $\mathrm{R}=0.498$ and $\mathrm{P}=0.0098$ (Eq. [7]).

\section{B.MP-MRI analysis}

From analysis of spatially registered multi-parametric MRI, the correlation coefficient $\mathrm{R}$ and $\mathrm{P}$ value for univariate fits between the eccentricity for the largest blob for varying thresholds (0.30 to 0.55) for ACE detection against the Gleason score (Eq. [6]) ranged from R -0.51 to -0.672 and $\mathrm{P}$ value from 0.0096 to 0.00023 for all 25 patients with positive Dynamic Contrast Enhancement-MRI scans and summarized in Table 2. Like the histology measurements in Table 1 and Reference 15, $b_{E c c}, t_{E c c}$, and $\mathrm{R}$ are negative using $\mathrm{ACE} / \mathrm{MP}-\mathrm{MRI}$ and implying that a more spherical tumor is more aggressive.

Metrics for assessing univariate fits to Gleason Score using the largest blob volume (Eq. [7]) following the application of thresholds 0.60 to 0.80 to ACE/MP-MRI are shown in Table 3. Specifically, the fitted coefficient (with its standard error), t-statistic (with the null-probability), R, $\mathrm{R}^{2}$ (with adjusted $\mathrm{R}^{2}$ ), F statistic (with null probability) are shown in Table 3. The correlation coefficients and t values are lower for ACE/MP-MRI volume measurements (Eq. [7]) than from ACE/MP-MRI eccentricity measurements (Eq. [6]) (Table 2), supporting the findings for the histologybased t-statistic magnitude of the eccentricity exceeds the volume t-statistic as shown in Table 1. Specifically, the $\mathrm{t}$ value $t_{E c c}$ in Table 2 ranges from -2.823 to -4.368 and the $\mathrm{t}$ value $t_{V o l}$ in Table 3 ranges from 2.807 to 2.468 .

The correlation coefficient for univariate fits between the largest blob volume for varying thresholds $(0.60$ to 0.80$)$ for ACE detection against the Gleason score (Eq. [7]) ranged from R 0.46 to 0.50 and $\mathrm{P}$ value 0.021 to 0.011 for all 25 patients with positive Dynamic Contrast EnhancementMRI scans. Figure $3 \mathrm{~A}$ plots the volumes of the largest blobs derived from different detection thresholds applied to ACE scores from MP-MRI against the total volume derived 
Table 3 Metrics for univariate fits to Gleason score using MP-MRI largest blob volume, thresholds 0.60 to 0.80

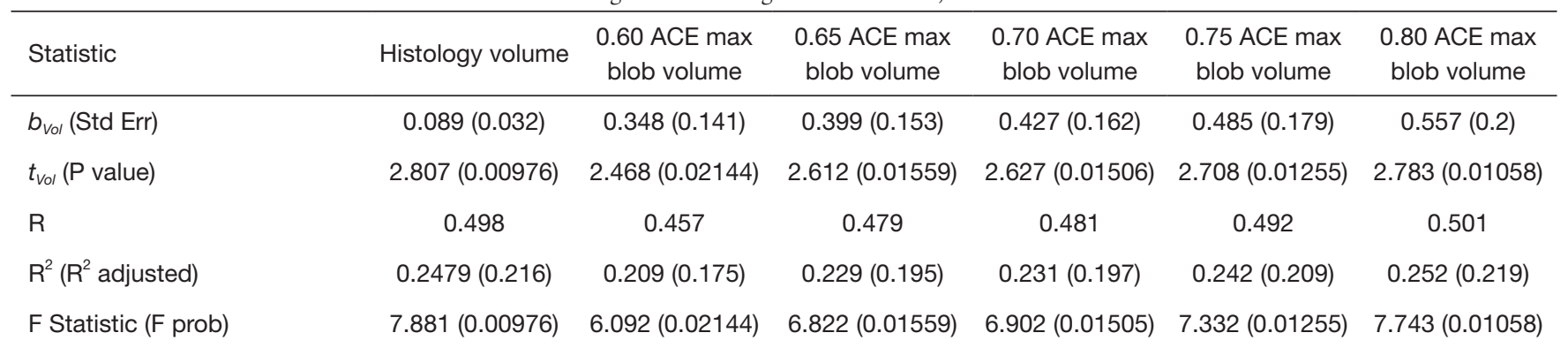

$b_{\text {Vol }}$, coefficient for volume; Std Err, standard error; $t_{\text {Vol }}, \mathrm{t}$ value for volume; $\mathrm{R}$, correlation coefficient; ACE max blob volume, volume of largest blob; $R^{2}$, squared correlation coefficient; $R^{2}$ adjusted, adjusted square correlation coefficient; ; $F$ prob, probability for $F$ statistic.
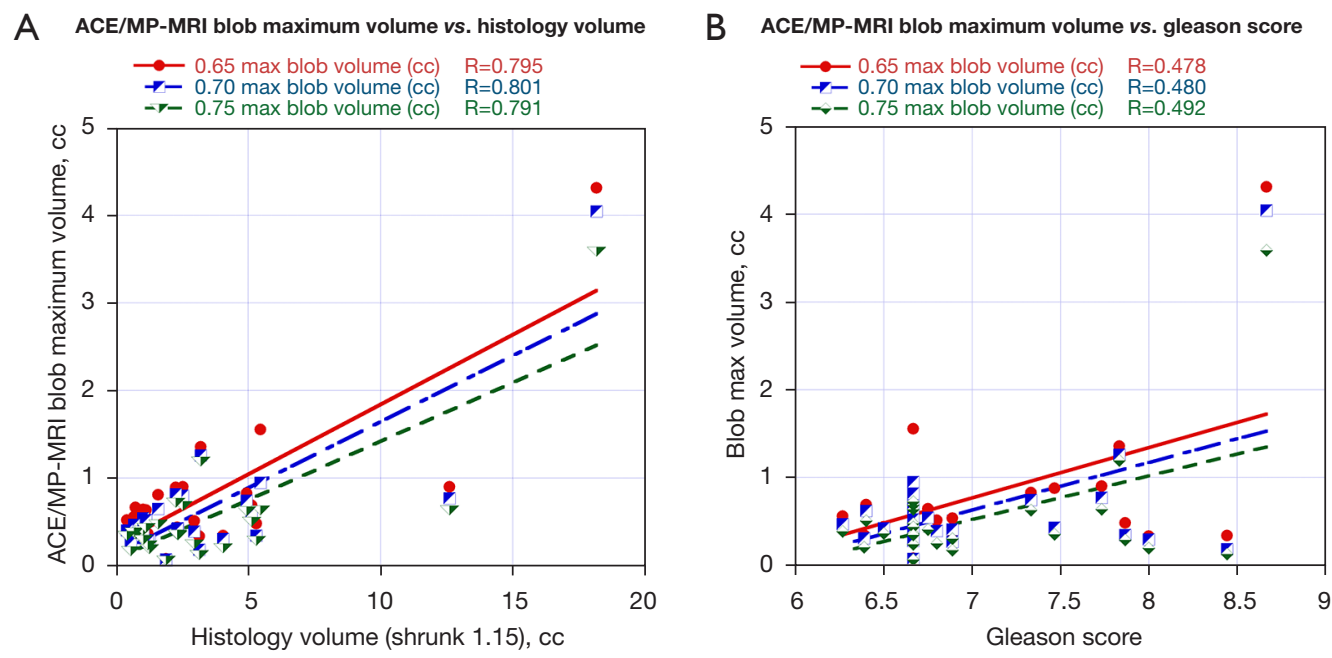

Figure 3 Scatterplots, correlation coefficients, and P values for fits of MP-MRI-based tumor volumes for 25 patients. (A) Plots the volumes of the largest blobs derived from different detection thresholds applied to ACE scores from MP-MRI against the total volume derived from histology; (B) plots the volumes of the largest blobs derived from different detection thresholds applied to ACE scores from MP-MRI against Gleason score.

from histology. The high correlation coefficients $\mathrm{R}$ range from 0.796 to 0.801 , validating volume measurements using ACE/MP-MRI and reconfirming the results from Reference (27). Figure $3 B$ plots the volumes of the largest blobs derived from different detection thresholds applied to ACE scores from MP-MRI against Gleason score (Eq. [7]). Lower correlation coefficients $\mathrm{R}(0.478$ to 0.492$)$ are calculated between the ACE/MP-MRI derived tumor volume and Gleason score (Eq. [7]) than the correlation coefficients computed for fits for curves in Figures $2 \mathrm{~B}, 3 \mathrm{~A}$.

Figure 4 plots the multivariate fit (Eq. [8]) using ACE/ MP-MRI (eccentricity for the largest blob with a 0.45 threshold plus volume for the largest blob with a 0.75 threshold) against data for Gleason score. The fit resulted in correlation coefficient of $\mathrm{R}=0.640$, lower than the univariate fit using only the eccentricity component (Eq. [6]).

\section{Discussion}

PCa management depends on reliable predictors of disease progression. Gleason score (7) is currently the most accurate independent variable for predicting PCa risk of biochemical recurrence, metastases, lymph node involvement, and seminal vesicle invasion following prostatectomy. Other independent variables such as tumor volume enhance prediction of disease progression through incorporation into nomograms of patient outcome. Due to its previous well researched history, Gleason score is used as a surrogate 


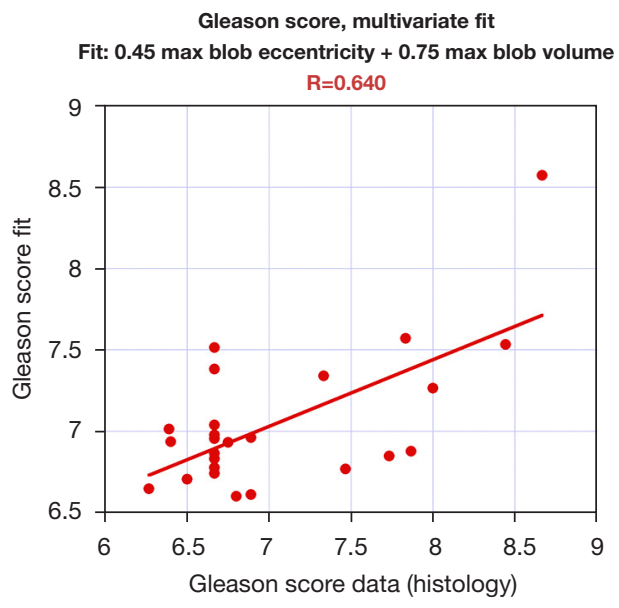

Figure 4 Plots the multivariate fit for Gleason score using MPMRI (0.45 ACE threshold, maximum blob eccentricity plus 0.75 ACE threshold, maximum blob volume) against data for Gleason score.

for disease progression for this effort. A significant result of this study, prostate tumor eccentricity, even better than the conventional tumor volume, correlates with Gleason score when measured using histology sections from prostatectomy and MP-MRI, and these findings support additional investigation to definitely establish the merits of tumor eccentricity as a standard factor for predicting outcome.

In fact, the relative magnitude of the t-statistic shows that eccentricity contributes more significantly for fitting the Gleason score than tumor volume. The magnitude of the t-statistics for eccentricity always exceeded the t-statistic for tumor volume in histology and MP-MRI multivariate fits. Conventionally, tumor volume is used to help stage PCa. However, this study suggests that eccentricity may play a more significant role in disease assessment, and as such could be considered for future revisions of the existing PCa staging system.

Tumor eccentricity derived from histology and MP-MRI correlated well with Gleason score. Tumor volume derived from histology correlated well with Gleason score and with volumes generated from MP-MRI. However, volumes derived from MP-MRI did not correlate well with Gleason score and added little to multivariate fits, unlike volumes taken from histology. The lack of improvement in fitting from multivariate fits using volumes from MP-MRI may result from the substantial difference in the better fitting using eccentricity from MP-MRI.

A note is added regarding MP-MRI-determined tumor volume and eccentricity measurements. The ACE detection thresholds for tumor volume were 0.60 to 0.80 and for eccentricity 0.30 to 0.55 . These thresholds were optimized to maximize the correlation of the independent variables (volume, eccentricity) to the dependent variable (Gleason scoring). Dynamic Contrast Enhancement forms a component of the target signature and, therefore, MPMRI generated tumor volume and eccentricity captures the angiogenic but not the poorly vascularized regions of tumors. As noted in earlier efforts (27), the MP-MRI tumor volume only captures one-third to half of the volume determined through histology but nevertheless correlates with the histology determined volume. Future work that excludes the DCE component may permit studies of tumor margins and its effect on clinical outcomes.

This study examined both histology specimens taken from prostatectomy and processed images derived from MP-MRI. Although analysis of prostatectomy specimens is often incorporated into disease projection models, a noninvasive prediction of disease outcome such as MRI would improve patient comfort and simplify and improve disease management. Nevertheless, tumor eccentricity and tumor volume measured through both methods may enhance currently limited prognostic information for patients and further guide early interventions to prevent or treat postprostatectomy recurrences.

Conventionally, Gleason score, PSA, tumor volume, lymph node involvement, and presence and location of metastases all contribute to predicting clinical outcomes, such as biochemical recurrence, for PCa. Adding tumor eccentricity, like other types of solid tumor cancers, may bolster PCa management.

There are a number of limitations for this study. While consecutive patients of a well-established dataset were used, the overall number of patient samples is limited, and all patients were assessed from a single institution. Future efforts should perform correlation studies and testing these algorithms with greater number of patient samples and across multiple institutions to increase clinical setting diversity and generalizability of our findings.

\section{Conclusions}

Prostate tumor eccentricity, determined either by histology or MP-MRI, more accurately predicted Gleason score than conventional prostate tumor volume from analysis of histology and MP-MRI. Combining tumor eccentricity and volume measurements derived from histology analysis 
further enhanced Gleason score prediction but was less successful using MP-MRI.

\section{Acknowledgments}

Funding: None.

\section{Footnote}

Reporting Checklist: The authors have completed the MDAR checklist. Available at https://dx.doi.org/10.21037/qims-21466

Conflicts of Interest: All authors have completed the ICMJE uniform disclosure form (available at https://dx.doi. org/10.21037/qims-21-466). The authors have no conflicts of interest to declare.

Ethical Statement: The authors are accountable for all aspects of the work in ensuring that questions related to the accuracy or integrity of any part of the work are appropriately investigated and resolved. The study was conducted in accordance with the Declaration of Helsinki (as revised in 2013). This retrospectively designed, single institution study was approved by the NIH Institutional Review Board, and was compliant with the Health Insurance Portability and Accountability Act. And individual consent for this retrospective analysis was waived.

Open Access Statement: This is an Open Access article distributed in accordance with the Creative Commons Attribution-NonCommercial-NoDerivs 4.0 International License (CC BY-NC-ND 4.0), which permits the noncommercial replication and distribution of the article with the strict proviso that no changes or edits are made and the original work is properly cited (including links to both the formal publication through the relevant DOI and the license). See: https://creativecommons.org/licenses/by-nc$\mathrm{nd} / 4.0 /$.

\section{References}

1. Shariat SF, Karakiewicz PI, Roehrborn CG, Kattan MW. An updated catalog of prostate cancer predictive tools. Cancer 2008;113:3075-99.

2. Martin NE, Mucci LA, Loda M, Depinho RA. Prognostic determinants in prostate cancer. Cancer J 2011;17:429-37.

3. Ho R, Siddiqui MM, George AK, Frye T, Kilchevsky
A, Fascelli M, Shakir NA, Chelluri R, Abboud SF, Walton-Diaz A, Sankineni S, Merino MJ, Turkbey B, Choyke PL, Wood BJ, Pinto PA. Preoperative Multiparametric Magnetic Resonance Imaging Predicts Biochemical Recurrence in Prostate Cancer after Radical Prostatectomy. PLoS One 2016;11:e0157313.

4. Wang L, Hricak H, Kattan MW, Chen HN, Kuroiwa K, Eisenberg HF, Scardino PT. Prediction of seminal vesicle invasion in prostate cancer: incremental value of adding endorectal MR imaging to the Kattan nomogram. Radiology 2007;242:182-8.

5. Shukla-Dave A, Hricak H, Kattan MW, Pucar D, Kuroiwa K, Chen HN, Spector J, Koutcher JA, Zakian KL, Scardino PT. The utility of magnetic resonance imaging and spectroscopy for predicting insignificant prostate cancer: an initial analysis. BJU Int 2007;99:786-93.

6. Poulakis V, Witzsch U, de Vries R, Emmerlich V, Meves M, Altmannsberger HM, Becht E. Preoperative neural network using combined magnetic resonance imaging variables, prostate-specific antigen, and gleason score for predicting prostate cancer biochemical recurrence after radical prostatectomy. Urology 2004;64:1165-70.

7. Gleason DF, Mellinger GT. Prediction of prognosis for prostatic adenocarcinoma by combined histological grading and clinical staging. J Urol 1974;111:58-64.

8. McNeal JE. Cancer volume and site of origin of adenocarcinoma in the prostate: relationship to local and distant spread. Hum Pathol 1992;23:258-66.

9. Villers AA, McNeal JE, Redwine EA, Freiha FS, Stamey TA. Pathogenesis and biological significance of seminal vesicle invasion in prostatic adenocarcinoma. J Urol 1990;143:1183-7.

10. Friedersdorff F, Groß B, Maxeiner A, Jung K, Miller K, Stephan C, Busch J, Kilic E. Does the Prostate Health Index Depend on Tumor Volume?-A Study on 196 Patients after Radical Prostatectomy. Int J Mol Sci 2017;18:488.

11. May M, Siegsmund M, Hammermann F, Loy V, Gunia S. Visual estimation of the tumor volume in prostate cancer: a useful means for predicting biochemical-free survival after radical prostatectomy? Prostate Cancer Prostatic Dis 2007;10:66-71.

12. McNeal JE, Villers AA, Redwine EA, Freiha FS, Stamey TA. Histologic differentiation, cancer volume, and pelvic lymph node metastasis in adenocarcinoma of the prostate. Cancer 1990;66:1225-33.

13. Epstein JI, Carmichael M, Partin AW, Walsh PC. Is tumor volume an independent predictor of progression following 
radical prostatectomy? A multivariate analysis of 185 clinical stage B adenocarcinomas of the prostate with 5 years of followup. J Urol 1993;149:1478-81.

14. Kikuchi E, Scardino PT, Wheeler TM, Slawin KM, Ohori M. Is tumor volume an independent prognostic factor in clinically localized prostate cancer? J Urol 2004;172:508-11.

15. Mayer R, Simone CB 2nd, Turkbey B, Choyke P. Correlation of prostate tumor eccentricity and Gleason scoring from prostatectomy and multi-parametricmagnetic resonance imaging. Quant Imaging Med Surg 2021;11:4235-44.

16. Moon HG, Kim N, Jeong S, Lee M, Moon H, Kim J, Yoo TK, Lee HB, Kim J, Noh DY, Han W. The Clinical Significance and Molecular Features of the Spatial Tumor Shapes in Breast Cancers. PLoS One 2015;10:e0143811.

17. Bae MS, Seo M, Kim KG, Park IA, Moon WK. Quantitative MRI morphology of invasive breast cancer: correlation with immunohistochemical biomarkers and subtypes. Acta Radiol 2015;56:269-75.

18. Yoon HJ, Park H, Lee HY, Sohn I, Ahn J, Lee SH. Prediction of tumor doubling time of lung adenocarcinoma using radiomic margin characteristics. Thorac Cancer 2020;11:2600-9.

19. Baba T, Uramoto H, Takenaka M, Oka S, Shigematsu Y, Shimokawa H, Hanagiri T, Tanaka F. The tumour shape of lung adenocarcinoma is related to the postoperative prognosis. Interact Cardiovasc Thorac Surg 2012;15:73-6.

20. Daniel Jensen J, Elewski BE. The ABCDEF Rule: Combining the "ABCDE Rule" and the "Ugly Duckling Sign" in an Effort to Improve Patient Self-Screening Examinations. J Clin Aesthet Dermatol 2015;8:15.

21. Ali AH, Li J, Yang G. Automating the ABCD Rule for Melanoma Detection: A Survey. IEEE Access 2020;8:83333-46.

22. King CR, Long JP. Prostate biopsy grading errors: a sampling problem? Int J Cancer 2000;90:326-30.

23. Epstein JI, Feng Z, Trock BJ, Pierorazio PM. Upgrading and downgrading of prostate cancer from biopsy to radical prostatectomy: incidence and predictive factors using the modified Gleason grading system and factoring in tertiary grades. Eur Urol 2012;61:1019-24.

24. Berglund RK, Masterson TA, Vora KC, Eggener SE, Eastham JA, Guillonneau BD. Pathological upgrading and up staging with immediate repeat biopsy in patients eligible for active surveillance. J Urol 2008;180:1964-7; discussion 1967-8.

25. Weinreb JC, Barentsz JO, Choyke PL, Cornud F, Haider
MA, Macura KJ, Margolis D, Schnall MD, Shtern F, Tempany CM, Thoeny HC, Verma S. PI-RADS Prostate Imaging - Reporting and Data System: 2015, Version 2. Eur Urol 2016;69:16-40.

26. Mayer R, Simone CB 2nd, Skinner W, Turkbey B, Choykey P. Pilot study for supervised target detection applied to spatially registered multiparametric MRI in order to non-invasively score prostate cancer. Comput Biol Med 2018;94:65-73.

27. Mayer R, Simone CB 2nd, Turkbey B, Choyke P. Algorithms applied to spatially registered multi-parametric MRI for prostate tumor volume measurement. Quant Imaging Med Surg 2021;11:119-32.

28. Choyke P, Turkbey B, Pinto P, Merino M, Wood B, Data From PROSTATE-MRI. The Cancer Imaging Archive. 2016. Available onlne: http://doi.org/10.7937/K9/ TCIA.2016.6046GUDv

29. Clark K, Vendt B, Smith K, Freymann J, Kirby J, Koppel P, Moore S, Phillips S, Maffitt D, Pringle M, Tarbox L, Prior F. The Cancer Imaging Archive (TCIA): maintaining and operating a public information repository. J Digit Imaging 2013;26:1045-57.

30. Shah V, Pohida T, Turkbey B, Mani H, Merino M, Pinto PA, Choyke P, Bernardo M. A method for correlating in vivo prostate magnetic resonance imaging and histopathology using individualized magnetic resonancebased molds. Rev Sci Instrum 2009;80:104301.

31. Turkbey B, Mani H, Shah V, Rastinehad AR, Bernardo M, Pohida T, Pang Y, Daar D, Benjamin C, McKinney YL, Trivedi H, Chua C, Bratslavsky G, Shih JH, Linehan WM, Merino MJ, Choyke PL, Pinto PA. Multiparametric $3 \mathrm{~T}$ prostate magnetic resonance imaging to detect cancer: histopathological correlation using prostatectomy specimens processed in customized magnetic resonance imaging based molds. J Urol 2011;186:1818-24.

32. Turkbey B, Pinto PA, Mani H, Bernardo M, Pang Y, McKinney YL, Khurana K, Ravizzini GC, Albert PS, Merino MJ, Choyke PL. Prostate cancer: value of multiparametric MR imaging at $3 \mathrm{~T}$ for detection-histopathologic correlation. Radiology 2010;255:89-99.

33. Tofts PS, Brix G, Buckley DL, Evelhoch JL, Henderson E, Knopp MV, Larsson HB, Lee TY, Mayr NA, Parker GJ, Port RE, Taylor J, Weisskoff RM. Estimating kinetic parameters from dynamic contrast-enhanced T(1)-weighted MRI of a diffusable tracer: standardized quantities and symbols. J Magn Reson Imaging 1999; 10:223-32.

34. Tofts PS, T1-weighted DCE Imaging Concepts: 
Modelling, Acquisition and Analysis. MAGNETOM Flash 2010;3:30-5.

35. Jain AK. Fundamentals of Digital Image Processing. Upper Saddle River, NJ: Prentice Hall; 1989.

36. Richards JA, Jia X. Remote Sensing Digital Image Analysis, New York: Springer-Verlag; 1999.

37. Manolakis D, Shaw G. Detection algorithms for hyperspectral imaging applications. IEEE Signal Process Mag 2002;19:29-43.

Cite this article as: Mayer R, Simone CB 2nd, Turkbey B, Choyke P. Prostate tumor eccentricity predicts Gleason score better than prostate tumor volume. Quant Imaging Med Surg 2022;12(2):1096-1108. doi: 10.21037/qims-21-466
38. Mardia KV, Kent JT, Bibby JM. Multivariate Analysis. Academic Press; 1979.

39. Jan K. Elements of Econometrics. 2nd ed. New York: Macmillan; 1986.

40. Walpole RE, Myers RH, Myers SL, Ye K, Yee K. Probability \& statistics for engineers \& scientists. Myers, H. Raymond. 7th ed. Prentice Hall College Div; 2002.

41. Chatterjee S, Simonoff J. Handbook of Regression Analysis. John Wiley Sons; 2013. 